\title{
Design and Implementation of Parking Sensing System Based on ZigBee Peng Guo ${ }^{1, a}$, Zhigang Wen ${ }^{2, b}$
}

\author{
${ }^{1}$ School of Electronic Engineering, Beijing University of Posts and Telecommunications, Beijing, \\ China \\ ${ }^{2}$ Institute of Sensing Technology and Business, BUPT, Wuxi, China \\ aguopengwell@163.com, bzwen@bupt.edu.cn
}

Keywords: RSSI; parking occupancy algorithm; parking sensing system

\begin{abstract}
With the development of parking lots towards large-scale and three-dimensional, intelligence has become an inevitable trend. In this paper, an intelligent parking lots detection system based on ZigBee transmission protocol is proposed. RSSI value is used to detect whether the parking lots are occupied. The system obtains parking information via ZigBee ad hoc network, data procession and transmission via parking detection gateway and users can obtain parking information via center management system at last.
\end{abstract}

\section{Introduction}

In order to improve our national's quality of life and quicken pace of life, private cars have become the first choice for many people travelling outside. Many cities with an average annual increase in the amount of cars result in car ownership is very huge, unsound vehicle management has brought many problems like parking difficulties and traffic jams. To some extend the emergence of various parking lots ease the shortage situation, but it also brings many problems of parking management. Therefore, with the development of parking lots towards large-scale and three-dimensional, intelligence has become an inevitable trend. As an important part of the smart parking lots, parking sensing system provide basic parking information for the system, this will make parking lots more efficient and intelligent.

In this paper, a new design of parking sensing management system is put forward. Data of parking spaces from the change of RSSI generated from ZigBee wireless communication.

\section{The architecture of parking sensing system}

Depend on the actual requirement, the architecture of the system is shown in Figure1

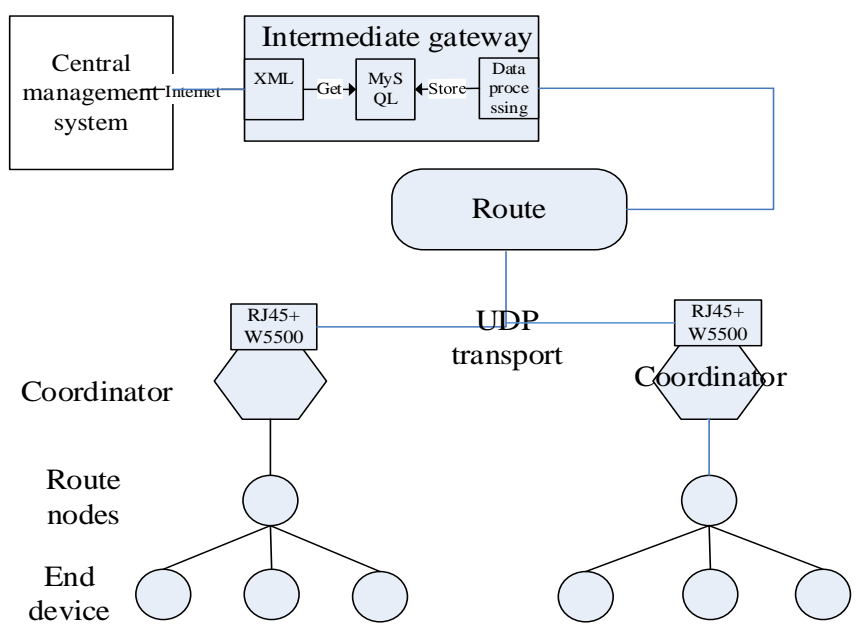

Fig.1. system architecture

The system is constituted by three subsystems, including parking space detection system, intermediate gateway and central management system. The status of parking spaces are determined 
by RSSI generated from communications between coordinators and end devices, and real-time status information of each parking space uploads to the central management system via intermediate gateway. According to these subsystems, this system can achieve real-time parking spaces status monitoring.

\section{Parking spaces detection system}

Parking space detection system is the data origin of the entire system. In general, intelligent parking lots have adopted many other detection schemes like infrared, ultrasonic, magnetic sensors and other sensor devices for data acquisition. And the defects of these sensor devices are also obvious, for example, magnetic sensors might be the most popular in these schemes, but its price is the most expensive almost thousands of RMB. Therefore, in this paper, a new method using the RSSI generated during ZigBee wireless communication is put forward. In low-power consumption, short-delay, near field, high capacity, low cost, ZigBee has its obvious advantages. What's more, this scheme do not require external sensors.

There's three types in a ZigBee network including end device, route and coordinator. Utilize the property of ZigBee ad hoc network, ZigBee end devices installed on the ground will send data to the ZigBee coordinators via ZigBee routes installed over the parking spaces. While the coordinators receive these messages, RSSI can be obtained. According to the change of this value, the parking spaces status can be analyzed.

In this detection system, there's also three parts: sender, relay and receiver. Adopt open-source hardware Arduino as MCU and other extension module like ZigBee and RJ45. The sender include ZigBee end device and the battery box, the relay include ZigBee router and battery module, the receiver include ZigBee coordinator, battery module, RJ45, W5500 and the MCU. In ZigBee network, the coordinator select an established channel and PANID to set up a network, and make the end devices and routes join in it. When end device first joins in the network, it will package its logical type, IEEE address and short address in a message, and send this message to the coordinator via route automatically. The message format is designed as following:

$+\mathrm{ZBC}=<$ logicalType $>$, $<$ localshortddr $>$, $<$ localieeeaddr $><\mathrm{CR}><\mathrm{LF}>$

Local short address is a unique network identifier distributed by coordinator, when coordinator receives this message indicating networking success.

When networking success, end devices and coordinators can pass messages to each other. When the messages in constant transmission, the received messages are structured as follows:

$+\mathrm{ZBD}=<$ localieeeddr $>$, $<\mathrm{RSSI}>*<\mathrm{CR}><\mathrm{LF}>$

For instance: $+\mathrm{ZBD}=500 \mathrm{D} 3 \mathrm{D} 05004 \mathrm{~B} 1200$, 045*. 500D3D05004B1200 is end device's IEEE address, a universally unique identifier. * is a delimiter.

When the senders are sending messages constantly, low-power consumption is necessary. After low-power processing, most of the connection time, ZigBee end devices are in a dormant status, and working current almost below $0.01 \mathrm{~mA}$, and during the extremely short period of transmission time, the working current varies between $0.2 \mathrm{~mA}$ and $0.8 \mathrm{~mA}$. Even if disconnected from the network, end devices are searching for the network to join, the current is also in a low level.

Theoretically, ZigBee coordinator can carry 65534 end devices, but it is not practical. In order to find the best deployment scheme for the network, many tests about antenna towards, types of cars, other parking spaces' impacts and other disturbances are executed. According to the typical results shown in Figure 2, the final deployment scheme is shown as Figure 3.

In Figure2, the above curve represents RSSI (-dB) when occupied, the below represents no car parked. The left shows parking space B's (in Figure3) RSSI, the right represents parking space A and C. If continue to add parking spaces besides A and C, the RSSI attenuation make it difficult to distinguish two kinds of status. In conclusion, using a route with three end devices is the ideal solution.

Coordinators ensure the channel and PANID of their own network, end devices and routes join their certain network. End devices send messages to corresponding with coordinator via route, receivers deal with these accepted messages and send them to gateway. Then through these efforts, 
a parking detection system is established.

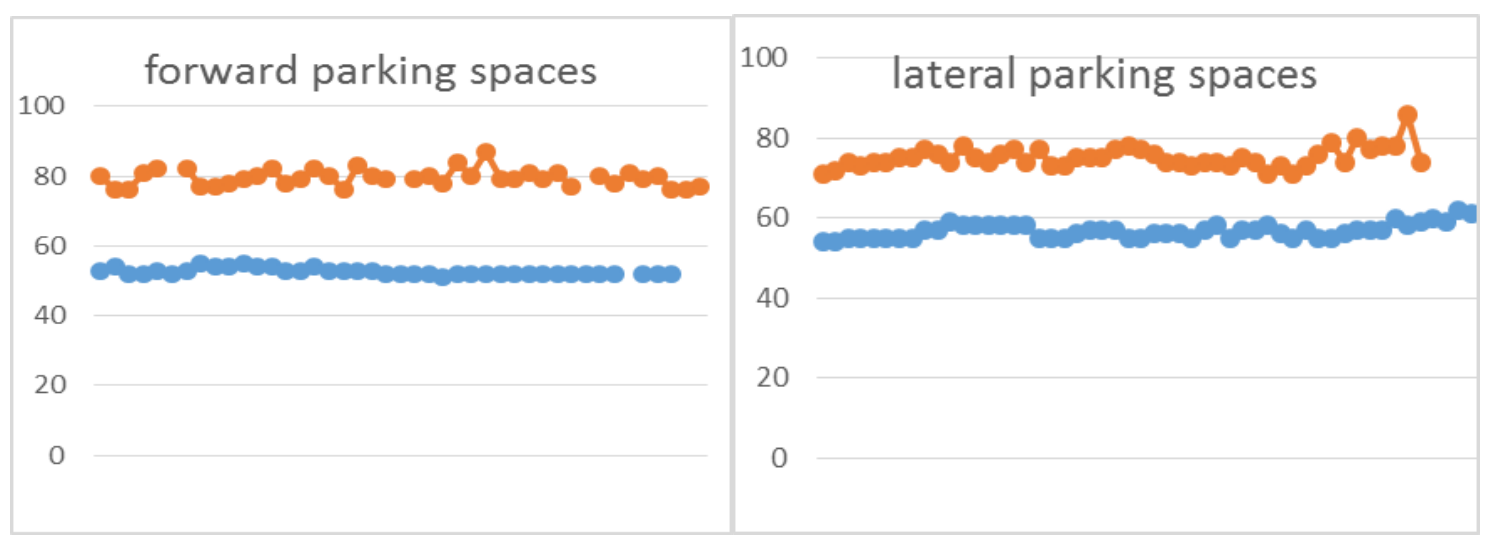

Fig.2. Test results

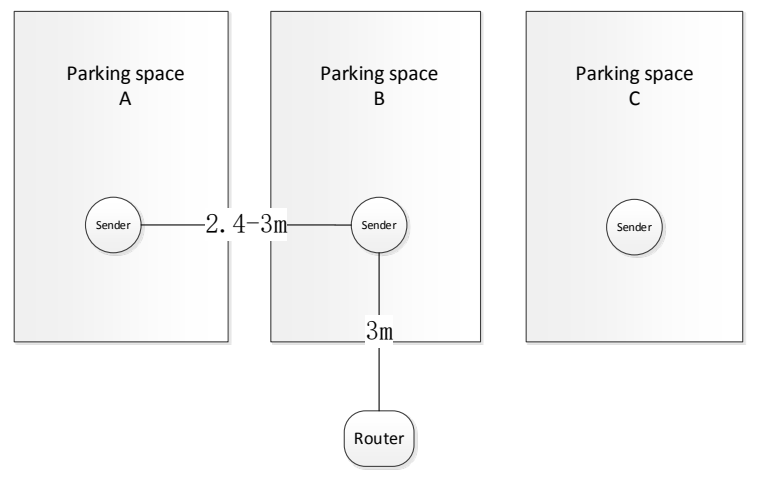

Fig.3. Deployment plan

\section{Intermediate gateway}

The function of intermediate gateway include processing messages received from parking spaces detection system and sending the processed data to the central management system.

According to the functions introduced above, the subsystem can be divided into two parts. One part of the role is to process message and store processed data into database, the other one is extracting date from database and packaging it into XML. This design can guarantee maximum independence of two modules and convenient maintain and redevelop.

\section{Algorithm for parking space occupancy}

Messages received by intermediate gateway contains IEEE address and RSSI. In Figure2, RSSI varies according to whether the vehicles is parked. Use the differences between two values can determine whether the space is occupied. In this module, IEEE address should be related to parking space ID as key and RSSI should be converted to parking space status (define number 0 is unoccupied and 1 is occupied) as value. Then a relationship of key-value is established.

Considering parking behavior is a long-term behavior, and RSSI fluctuated by other uncertain interferences. Therefore, a given moment cannot be used to judge the status. So an algorithm for parking space occupancy is designed. Algorithm is designed into two areas: determine threshold value and eliminate noise.

Randomness of the wireless channel, surroundings, battery capacity decrease and other factors may influence the decision of the threshold. Set a time interval (this time should be longer), register the RSSI of this period, analyze the average value of occupied and unoccupied, and then compute the average of the two values as threshold. To some extent, an obvious threshold can be classified as Figure2. Repeat the above process continuously and this value will be adjusted automatically. This design is mainly used to meet the demand of different environment and in case of battery capacity decrease.

The threshold describe the variation in a long period of time, the current parking status should be determined by comparing current RSSI with the threshold. But uncertain noises may cause RSSI 
a sudden change and influence the result. For purpose of eliminating these noises, algorithm is designed to imitate the character of Markov Chain, the value of current state depends on the previous finite state.

Calculate the average of the last $n$ received RSSI, compare it with the current threshold, and then store this comparison result into an intermediate variable named temp. And use the character of hash map, the key-value relationship, the key represents ID and the value always stores the temp of previous state. If current temp equals to the previous temp in hash map, then the result does not change. Else if two temps are not equal, then put current temp into hash map and wait for next state, if the next temp equals to the previous and not equals to the current, then the result does not change, else if the next temp equals the current, the result change to another status. Example as following table:

Tab.1. Algorithm example

\begin{tabular}{|l|c|c|c|c|c|c|c|c|}
\hline Time node & $3 \mathrm{~s}$ & $6 \mathrm{~s}$ & $9 \mathrm{~s}$ & $12 \mathrm{~s}$ & $15 \mathrm{~s}$ & $18 \mathrm{~s}$ & $21 \mathrm{~s}$ & $\ldots$ \\
\hline temp & 0 & 0 & 1 & 0 & 1 & 1 & 1 & $\ldots$ \\
\hline outcome & 0 & 0 & 0 & 0 & 0 & 1 & 1 & $\ldots$ \\
\hline
\end{tabular}

In this chart, at node 9s, there is a mutant occurring, but the outcome does not change. And at $15 \mathrm{~s}$, the change occurs at $18 \mathrm{~s}$.

\section{Data sending}

After data processing, the data stores into the database. Only after sending data to the central management system, the data becomes meaningful. Design as REST, the interface can implement data sharing. Not only central management can receive this message, but also the third parties can use this resource.

The data are encapsulated into XML as following:

$<$ AirPortParkingManage $>$

$<$ sensor_code $>$ parking ID $<$ /sensor_code $>$

$<$ occupy $>$ parking status $<$ /occupy $>$

$<$ data_time $>$ time $</$ data_time $>$

$</$ AirPortParkingManage $>$

The central management system receives the XML, then parses the XML, and at last obtain the useful informations.

\section{Central management system}

There is two main functions of this system, one is resources open to the platform by the interaction between the intermediate gateway and central system. The second is through the central management system implements the resources management, open sources by the REST interface, sharing resources.

Data sending has been implemented by the intermediate gateway, XML can be received by the central system as feed. Figure 4 is the feed received by central system.

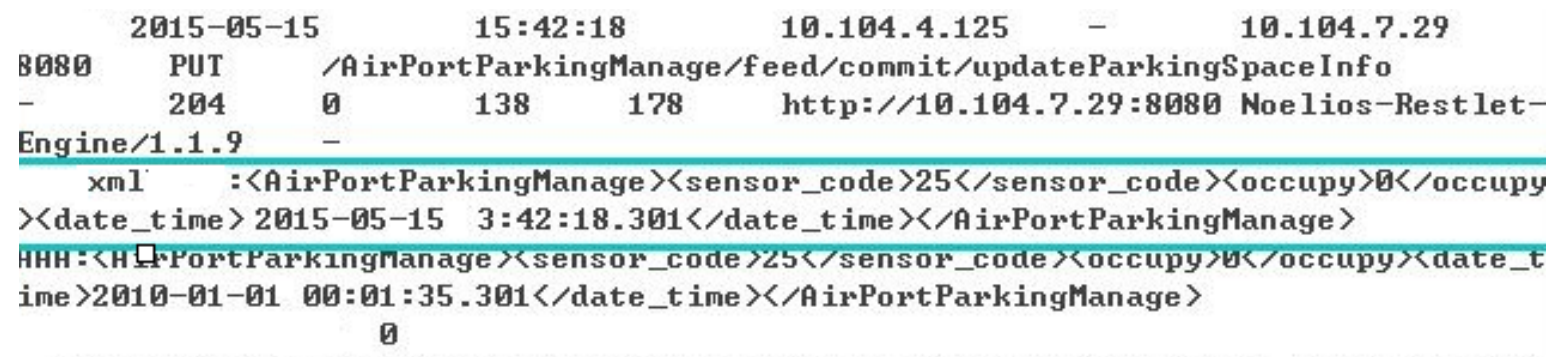

jdbc : mysq $1: / /$ localhost $: 3306 /$ parkingmanage?characterEncoding =utf -8 , UserName $=$ root llocalhost, MySQL-AB JDBC Driver

Fig.4. Feed information 
Through the REST interface, central management system can open its parking status information. Through this interface, the external application can obtain the status of parking resources. At this point can realize the Internet of Things.

\section{Conclusion}

Based on the design of the system as the foundation, a demonstration project is deployed in an indoor parking lot. And after measuring its accuracy, limited by the experimental conditions, the system can able to detect most of the parking spaces, nearly about ninety percent. But this can be improved by the industrial production. At least this project proves the feasibility of this system. This system realizes another scheme about parking sensing system, it has the advantages on price and convenient deployment. The most important is its accuracy can also be ensured.

This system also has its weakness. Since the use of the variation on RSSI blocked by vehicles, the route should be installed on the top of the parking spaces, so this system should only be deployed in indoor parking lots.

\section{Acknowledgment}

The work presented in this paper was supported by the National Great Science Specific Project (Grants No. 2014ZX03002002-004) and National Natural Science Foundation of China (Grants No. NSFC-61471067).

\section{References}

[1] Jiameng Wang; Gebara, S.; Zhengyi Sun; Qi Wu; Keqin Zong; Hao Sun; Farajidavar, A., "IPLMS: An intelligent parking lot management system," in Systems, Applications and Technology Conference (LISAT), 2015 IEEE Long Island , vol., no., pp.1-6, 1-1 May 2015

[2] Yucheng Li; Guohui Li; Xingcai Zhao, "Approach for Parking Spaces Detection Based on ARM Embedded System," in Intelligent Human-Machine Systems and Cybernetics (IHMSC), 2013 5th International Conference on , vol.2, no., pp.406-409, 26-27 Aug. 2013

[3] Cook, D.J.; Morris, T.; Morellas, V.; Papanikolopoulos, N., "An automated system for persistent real-time truck parking detection and information dissemination," in Robotics and Automation (ICRA), 2014 IEEE International Conference on , vol., no., pp.3989-3994, May 31 2014-June 7 2014

[4] Jae Kyu Suhr; Ho Gi Jung, "Sensor Fusion-Based Vacant Parking Slot Detection and Tracking," in Intelligent Transportation Systems, IEEE Transactions on , vol.15, no.1, pp.21-36, Feb. 2014

[5] Suzuki, N.; Mitani, T.; Shinohara, N., "Study and development of a microwave power receiving system for ZigBee device," in Microwave Conference Proceedings (APMC), 2010 Asia-Pacific , vol., no., pp.45-48, 7-10 Dec. 2010

[6] Firdaus; Nugroho, E.; Sahroni, A., "ZigBee and wifi network interface on Wireless Sensor Networks," in Electrical Engineering and Informatics (MICEEI), 2014 Makassar International Conference on , vol., no., pp.54-58, 26-30 Nov. 2014 